\title{
Magnet Formation by the Surface Modification of Diamond with Manganese Detected by the Magnetic Flux Density on the Surface
}

\author{
Takahisa Yamazaki, Ryusei Ninomiya \\ School of Engineering, Tokyo Institute of Technology, Tokyo, Japan \\ Email: yamazaki.t.ae@m.titech.ac.jp
}

How to cite this paper: Yamazaki, T. and Ninomiya, R. (2017) Magnet Formation by the Surface Modification of Diamond with Manganese Detected by the Magnetic Flux Density on the Surface. Materials Sciences and Applications, 8, 642-648.

https://doi.org/10.4236/msa.2017.88045

Received: May 22, 2017

Accepted: August 14, 2017

Published: August 17, 2017

Copyright ( 92017 by authors and Scientific Research Publishing Inc. This work is licensed under the Creative Commons Attribution International License (CC BY 4.0). http://creativecommons.org/licenses/by/4.0/

\begin{abstract}
A surface modification of diamond with manganese powder was attempted to make a magnetic functional surface for handling diamond. Manganese carbide, $\mathrm{Mn}_{7} \mathrm{C}_{3}$, was formed on diamond (110) by near-infrared radiation heating, resulting in a layered material with suppressing formation of $\mathrm{Mn}_{5} \mathrm{C}_{2}$. Investigated by a Hall-effect sensor, the magnetic flux density on the modified diamond surface showed ferromagnetic properties.
\end{abstract}

\section{Keywords}

Manganese, Diamond, Carbide, Magnet, Hall-Effect Sensor

\section{Introduction}

Diamond has excellent properties of hardness, compressive strength, electric insulation, and chemical stability [1]. Surface modification of diamond is an effective method to improve its wettability toward metals for industrial use [2]. Fine-grain diamond has been used as an abrasive powder for materials, however, they were not easily placed into particular areas to serve as blades. If diamond surface could be modified to have magnetic properties, we could evaluate the potential for the surface serve as a magnetic sensor and the damage of diamond.

We previously reported that manganese powder reacts with carbon atoms on diamond, forming manganese carbides and manganese oxides. The reaction only modified diamond (110) plane, providing ferrimagnetism at room temperature and the formation of layered manganese carbides [3]. Karen et al. [4] characterized the magnetic properties of $\mathrm{Mn}_{5} \mathrm{C}_{2}$ and $\mathrm{Mn}_{7} \mathrm{C}_{3}$ bulk manganese carbides as paramagnetic. We believe that the modification of manganese powder along the 
specific diamond plane producing nanoscopic layers provides spontaneous magnetization. Using infrared radiation heating, the surface of diamond decomposes by the molecular vibrations, desorbing carbon dioxide gas and hydrocarbons from the surface [5].

Zhang et al. [6] reported six electromagnets set in a regular hexagonal apex construction, where the top surface of the cylinder exhibited magnetic levitation using a Hall-effect sensor. We attempted to detect the magnetic flux from 6 manganese carbide products set in hexagonal apexes on the diamond (110) surface by Hall-effect sensor.

\section{Experimental Procedures}

\subsection{Preparation of Diamond (110)}

Single crystal diamond was processed by machining with an abrasive diamond grain and cleaning with acetone. The exposed face of diamond sample was (110) plane. Surface modification was processed as following: sputtering of gold coating (99.9\% purity) by an argon ion beam on the diamond was conducted to prevent the reaction with manganese by smoothing a gold film of $60 \mathrm{~nm}$ in thickness. At six specific spots, as shown in Figure 1, the gold film was peeled off the diamond (110) surface under an optical microscope. Manganese powder with an average diameter of less $100 \mu \mathrm{m}$ ground by ceramic ball milling to have a diameter less than $45 \mu \mathrm{m}$.

A bulk sample of diamond (natural type Ia, dimensions: $1.4 \times 5.0 \times 2.0 \mathrm{~mm}$ ) was placed in a platinum cell, and a small amount of manganese powder was mounted on diamond (110). The specimens were irradiated with near-infrared radiation using an elliptic mirror with a quartz viewing port, as shown in Figure 2. The spot diameter was $18 \mathrm{~mm}$, and it was focused on the manganese powder. Diamond is transparent to near-infrared radiation, so the input heat was restricted to the surface of the diamond-mounted manganese powder. An ultrahigh vacuum chamber was used to prevent the oxidation of manganese, which was tracked by the differential thermal analysis under argon gas and X-ray diffraction patterns to detect thermal increases after heating over $670 \mathrm{~K}$ and the formation of $\mathrm{Mn}_{2} \mathrm{O}_{4}$ and $\mathrm{MnO}$, based on the results of previous study. A ther-

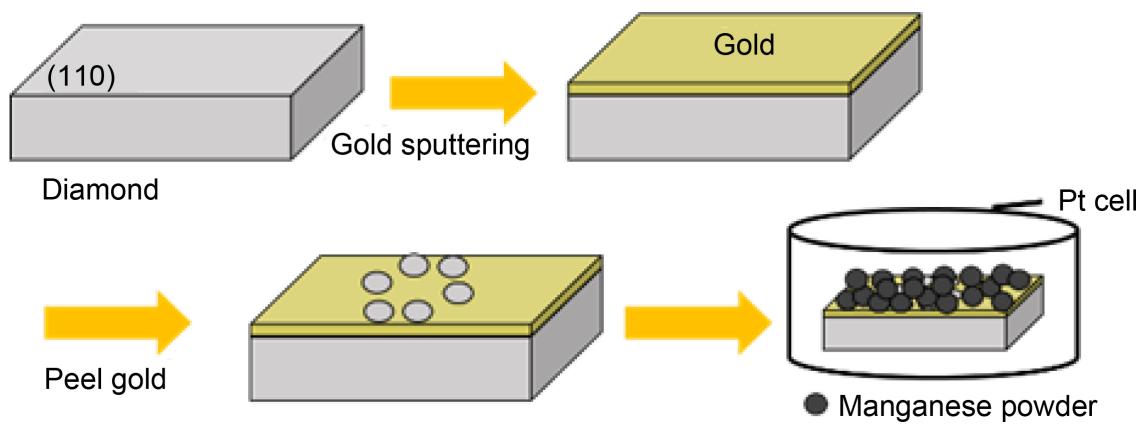

Figure 1. Schematic illustration of the processing of diamond (110) covered with a gold film. 


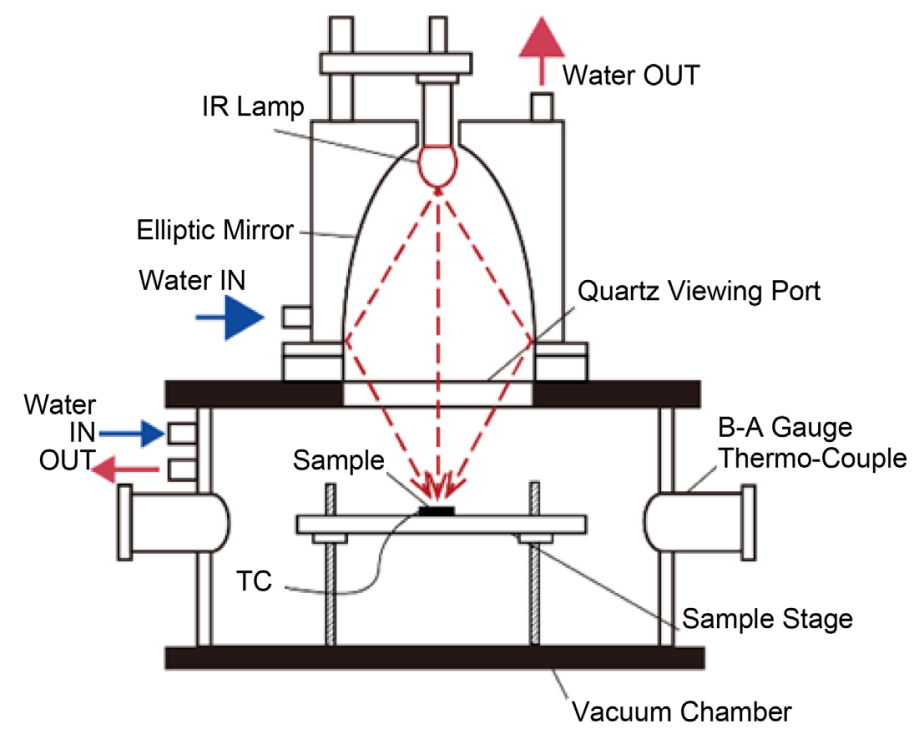

Figure 2. Schematic illustration of infrared heating of the device.

mocouple was fixed on a quartz stage to make contact to the bottom of the platinum cell to measure its temperature avoiding direct infrared radiation. The mixture of diamond powder and manganese powder exothermically reacted at $693 \mathrm{~K}$, as detected the differential thermal and gravitational analyses. The modification process was conducted under vacuum at $8 \times 10^{-4} \mathrm{~Pa}$ at $623 \mathrm{~K}$.

$\mathrm{X}$-ray diffraction analysis was performed on the modified diamond surface to detect the carbide reaction products.

\subsection{Magnetic Flux Measurements}

Magnetic flux measurement was performed on the modified diamond surface and non-modified diamond surface to assess spontaneous magnetization using a Hall-effect sensor at room temperature and low temperature. The specifications of the Hall-effect sensor specification are listed in Table 1. The sensor was applied with $1.78 \mathrm{~V}$ and $250 \mathrm{~mA}$, as shown in the measurement circuit. The output signal from the sensor was amplified by a thousand times.

\section{Results}

\subsection{Modified Diamond (110) by Manganese}

The modified diamond (110) specimen is shown in Figure 3. It became black in color after modification, and hexagonal formations were observed. The spots are numbered clock wise from 1 to 6 . The hexagons with metallic luster were observed. This reveals that the manganese powder directly contacted with diamond was different from the one contacted with the gold film. The X-ray diffraction pattern of the modified diamond (110) is shown in Figure 4. The peak of diamond (110) at $75^{\circ}$ is observed. The peaks of pure manganese at $42.9^{\circ}$ and $48^{\circ}$ are observed, and pure gold peaks at $39^{\circ}$ and $65^{\circ}$ are also observed. Peaks of manganese carbides, $\mathrm{Mn}_{5} \mathrm{C}_{2}$ at $44.8^{\circ}, 45.4^{\circ}$ and $49.7^{\circ}$ are observed. These peaks 
Table 1. Specifications of the Hall-effect sensor.

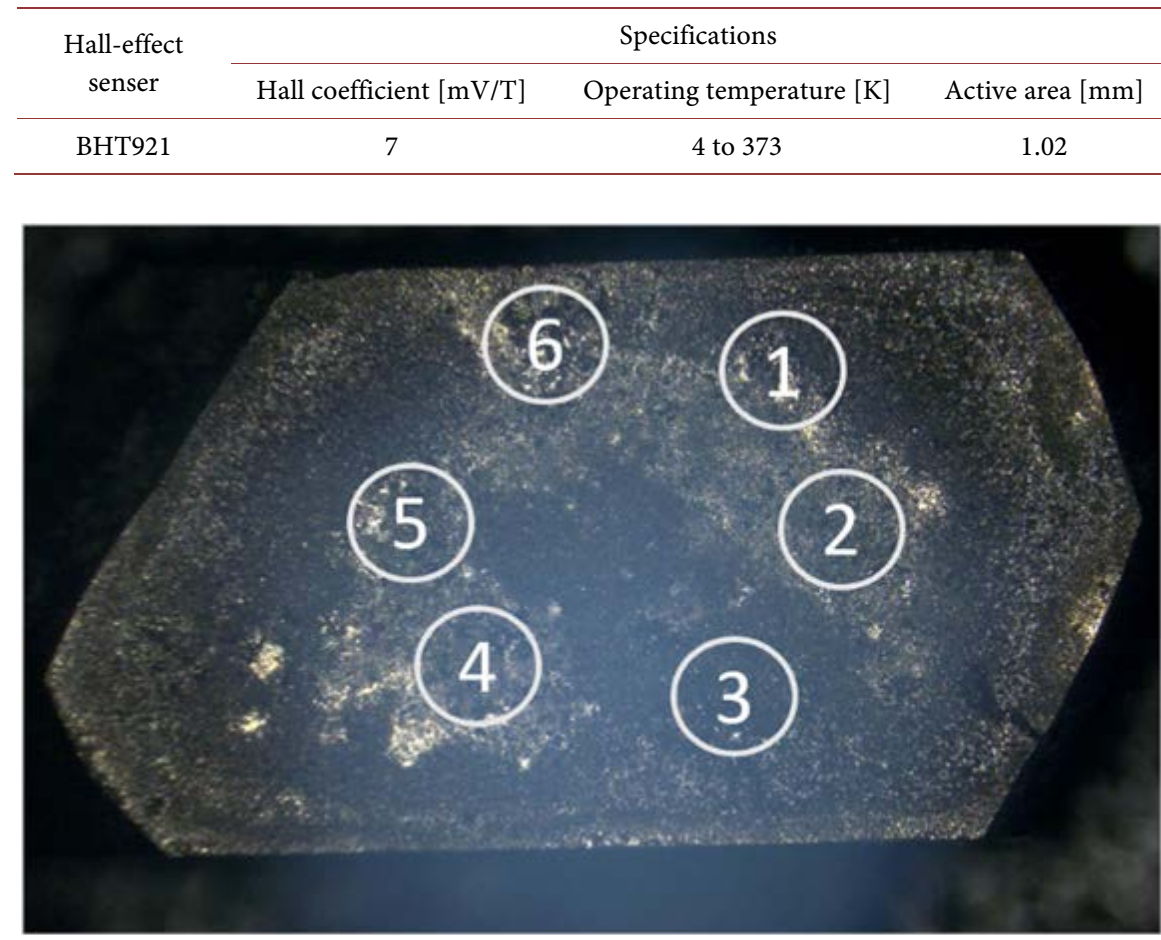

Figure 3. Appearance of the modified surface and the hexagonal spots showing the magnet formation.

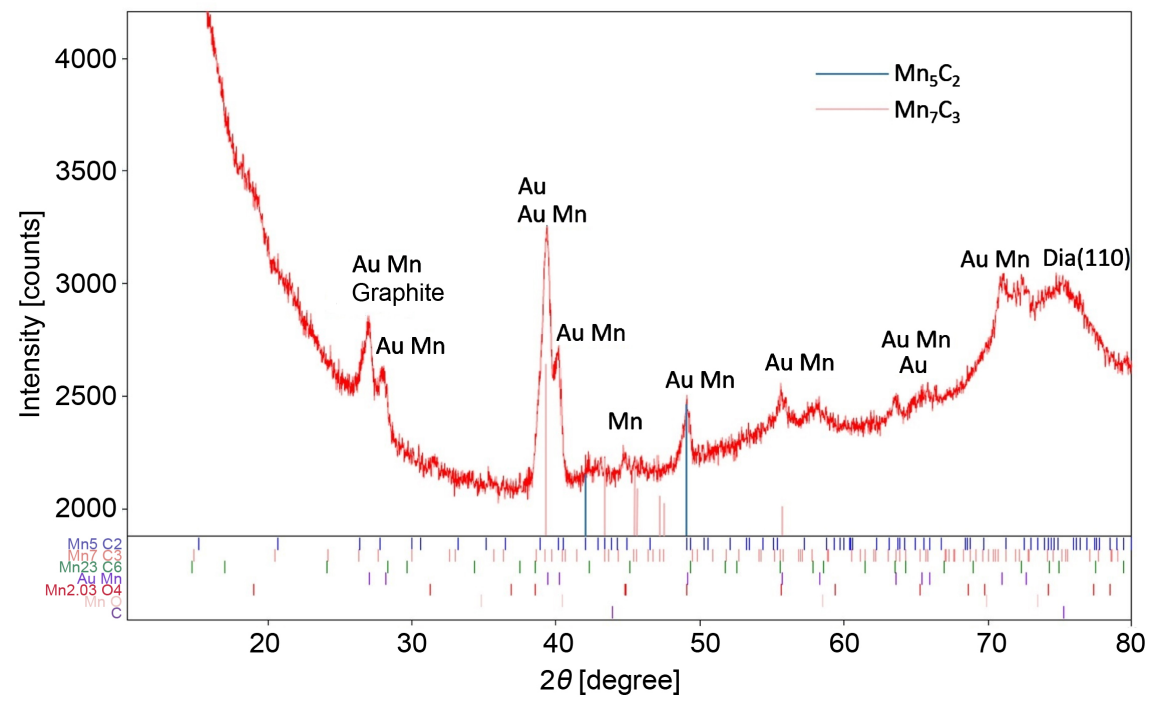

Figure 4. X-ray diffraction pattern after the surface modification of diamond (110) with the manganese powder.

are overlapped with gold-manganese peaks. Peaks of $\mathrm{Mn}_{7} \mathrm{C}_{3}$ at $39.5^{\circ}, 42.5^{\circ}$ and $44.6^{\circ}$ are observed. A peak of manganese oxide cannot be observed.

\subsection{Measurement of Magnetic Flux at Various Temperatures}

Figure 5 shows the results of the measured magnetic flux densities on spot A 


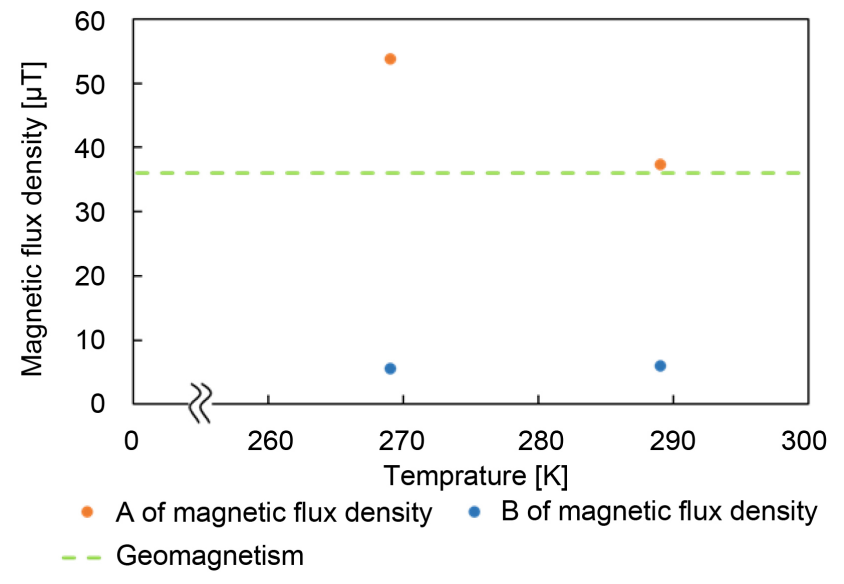

Figure 5. Magnetic flux densities on the modified diamond (110) with remaining gold film and with the gold film removed.

(number 3), where manganese carbides formed, and on spot B, which was covered with gold to distinguish the carbide magnet formation with varied temperature. Spot B was outside the hexagon near the diamond edge. The difference of the measured magnetic flux density at the two locations was apparent. The magnetic flux density on gold covered area was negligibly small $(5 \mu \mathrm{T})$, and a temperature dependence was not observed. On the other hand, the modified (110) surface with manganese powder exhibited the magnet properties. The magnetic flux density increased greater than that of geomagnetism, as shown in the broken line upon lowering the temperature.

\subsection{Detection of the Center by a Hall-Effect Sensor}

In addition, using the active area of the Hall-effect sensor $(1.02 \mathrm{~mm}$ in diameter), measurements of the magnetic flux densities on the hexagonal spots numbered from 1 to 6 were conducted at a low temperature of $262 \mathrm{~K}$. The results of the measurements at $262 \mathrm{~K}$ are presented in Table 2. The amount of formed manganese carbide was different in each spots, and therefore, the measurement values were also different. The direction of the spontaneous magnetism was aligned. The temperature of $262 \mathrm{~K}$ was detected by the thermocouple on the chilled copper foil dipped into liquid nitrogen on the other side.

Since diamond was covered with gold, the modified diamond was moved on top of the Hall device, which was near the manganese carbide for the Hall sensor measurement. The magnetic flux densities were measured along diagonal lines. The maximum magnetic flux density was measured near the center of the hexagonal spots, near spot 3 . The measured magnetic flux density was $73 \mu \mathrm{T}$.

\section{Discussion}

In our previous study, diamond (110) modified by manganese provided ferromagnetic properties as shown in Figure 6, which shows the relationship between the magnetic flux density and the magnetic field at room temperature measured 
Table 2. Magnetic flux densities measured at the six spots.

\begin{tabular}{ccccccc}
\hline & \multicolumn{7}{c}{ Location } \\
\cline { 2 - 7 } & 1 & 2 & 3 & 4 & 5 & 6 \\
\hline $\begin{array}{c}\text { Magnetic flux } \\
\text { density }[\mu \mathrm{T}]\end{array}$ & 28 & 37 & 51 & 28 & 45 & 40 \\
\hline
\end{tabular}

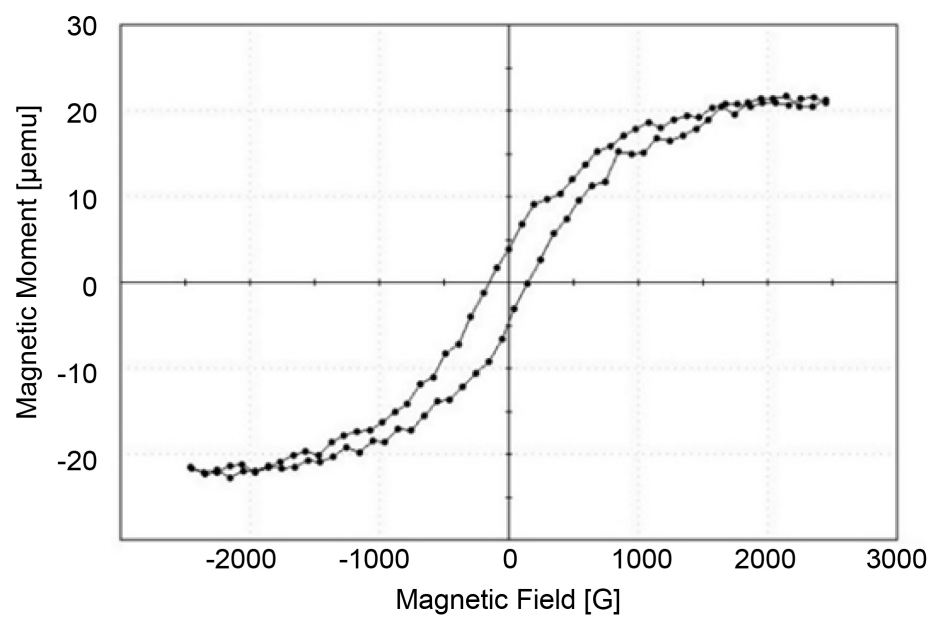

Figure 6. B-H curves of the manganese carbides on diamond (110) [2].

by a vibration sample magnetometer (VSM). The magnetic moment $4 \mu \mathrm{emu}$ $\left(\mathrm{mA} \cdot \mathrm{m}^{-1}\right)$ was smaller than those of the 6 spots in this work because the bottom surface was also modified with manganese and weaken the magnetic moment of the top surface. The magnetic moment originating from the sectional spots without gold coating became large due to the small amount of manganese carbides. In this study, the modified diamond surface processed spontaneous magnetism, forming layered manganese carbides. For the magnetized layer, the magnetic flux density was greater than that at lower temperature.

Surveying with the Hall-effect sensor along diagonal lines of the hexagon spots revealed the center of a hexagon on diamond (110). This indicates that diamond modified with manganese can be precisely controlled by magnetic force.

\section{Conclusion}

A diamond surface was modified with a manganese powder using near-infrared heating at $623 \mathrm{~K}$. The X-ray diffraction pattern showed the formation of manganese carbides of $\mathrm{Mn}_{7} \mathrm{C}_{3}$ and $\mathrm{Mn}_{5} \mathrm{C}_{2}$. The diamond surface covered with gold did not react with manganese powder, and only six spots where gold was removed reacted with manganese under vacuum at a pressure of $8 \times 10^{-4} \mathrm{~Pa}$ (to prevent oxidation) by heating with near-infrared radiation. Layered manganese carbides formed on diamond (110) by the radiation heating at $693 \mathrm{~K}$. The measured magnetic flux density from the modified diamond (110) by a Hall-effect sensor was greater than that of geomagnetism, with a maximum value of $73 \mu \mathrm{T}$ at the 
center of the diagonal line between hexagonal spots 1 - 3. It would be a magnetic sensor of damage of diamond.

\section{Acknowledgements}

This research was supported by the Center for Advanced Materials Analysis at the Tokyo Institute of Technology for X-ray Diffraction. The research was also supported by the Japan Society for Promotion of Science KAKENHI (23656452).

\section{References}

[1] Novicov, H.B. (Editor) (1993) Physical Property of Diamond, Ohm Printing in Japan.

[2] Yu, L., Ostrovskaya, A.P., Dementiev, I.I., Kulakova and Ralchenko, V.G. (2005) Chemical State and Wettability of Ion-Irradiated Diamond. Diamond and Related Materials, 14, 486-490.

[3] Tsuda, H., Yamazaki, T., Ikeshoji, T.-T. and Suzumura, A. (2013) Change of Magnetic Properties on Modified Diamond Surface with Manganese Powder. Proceedings of IJST 2013, Osaka, 27-29 November 2013, SPI-1.

[4] Kjekshus, K.A., Anderson, A.F. and Fjellvag, H. (1991) On the Phase Relations Structural Relations and Structural and Magnetic Properties of the Stable Manganese Carbide, Mn23C6, Mn5C2, Mn7C3. Acta Chemica Scandinavica, 45, 549-557. https://doi.org/10.3891/acta.chem.scand.45-0549

[5] Yamazaki, T., Tsuda, H. and Ikeshoji, T.-T. (2013) A Suzumura, Reaction Product between Diamond and Manganese Powder Heated by Condensed Infrared Ray Formation, Brazing, High Temperature Brazing and Diffusion Bonding, Lot 2013, DVS 293, 283-286.

[6] Zhang, X.D., Mehrtash, M. and Khamesee, M.B. (2015) Dual-Axial Motion Control of a Magnetic Levitation System Using Hall-Effect Sensors. IEEE/ ASME Transactions on Mechatronics, 2015, 1-11. https://doi.org/10.1109/TMECH.2015.2490118

Submit or recommend next manuscript to SCIRP and we will provide best service for you:

Accepting pre-submission inquiries through Email, Facebook, LinkedIn, Twitter, etc. A wide selection of journals (inclusive of 9 subjects, more than 200 journals) Providing 24-hour high-quality service User-friendly online submission system Fair and swift peer-review system Efficient typesetting and proofreading procedure Display of the result of downloads and visits, as well as the number of cited articles Maximum dissemination of your research work

Submit your manuscript at: http://papersubmission.scirp.org/

Or contact msa@scirp.org 